\title{
A Hybrid Electrical/Chemical Circuit in the Spinal Cord Generates a Transient Embryonic Motor Behavior
}

\author{
(ㄴ)Laura D. Knogler, ${ }^{1,2 \star}$ Joel Ryan, ${ }^{1 \star}$ Louis Saint-Amant, ${ }^{1}$ and Pierre Drapeau ${ }^{1,2}$ \\ Departments of ${ }^{1}$ Pathology and Cell Biology and ${ }^{2}$ Neuroscience, Research Centre of the University of Montréal Hospital Centre and Groupe de Recherche \\ sur le Systeme Nerveux Central, Université de Montréal, Montréal, Québec H3T 1J4, Canada
}

\begin{abstract}
Spontaneous network activity is a highly stereotyped early feature of developing circuits throughout the nervous system, including in the spinal cord. Spinal locomotor circuits produce a series of behaviors during development before locomotion that reflect the continual integration of spinal neurons into a functional network, but how the circuitry is reconfigured is not understood. The first behavior of the zebrafish embryo (spontaneous coiling) is mediated by an electrical circuit that subsequently generates mature locomotion (swimming) as chemical neurotransmission develops. We describe here a new spontaneous behavior, double coiling, that consists of two alternating contractions of the tail in rapid succession. Double coiling was glutamate-dependent and required descending hindbrain excitation, similar to but preceding swimming, making it a discrete intermediary developmental behavior. At the cellular level, motoneurons had a distinctive glutamate-dependent activity pattern that correlated with double coiling. Two glutamatergic interneurons, CoPAs and CiDs, had different activity profiles during this novel behavior. CoPA neurons failed to show changes in activity patterns during the period in which double coiling appears, whereas $\mathrm{CiD}$ neurons developed a glutamate-dependent activity pattern that correlated with double coiling and they innervated motoneurons at that time. Additionally, double coils were modified after pharmacological reduction of glycinergic neurotransmission such that embryos produced three or more rapidly alternating coils. We propose that double coiling behavior represents an important transition of the motor network from an electrically coupled spinal cord circuit that produces simple periodic coils to a spinal network driven by descending chemical neurotransmission, which generates more complex behaviors.
\end{abstract}

Key words: glutamate; locomotor development; motoneurons; spinal cord; V2a interneurons; zebrafish embryo

\section{Introduction}

Spontaneous network activity is a highly stereotyped feature of developing circuits throughout the nervous system, including the spinal cord (Feller, 1999; Blankenship and Feller, 2010). Spinal locomotor circuits produce a series of behaviors during development that reflect the continual integration of spinal neurons into a functional network. In the embryonic spinal cord, the first motor activity is a slow coiling of the trunk that is sensoryindependent and therefore appears to be centrally driven (Hamburger, 1963; Narayanan et al., 1971; Corner, 1978). Embryos later become responsive to sensory stimuli and eventually locomote, behaviors that are shaped by the integration of central patterns and sensory feedback (van Mier et al., 1989; Dale, 1995).

Received March 26, 2014; revised May 12, 2014; accepted June 8, 2014.

Author contributions: L.D.K., J.R., L.S.-A., and P.D. designed research; L.D.K. and J.R. performed research; L.D.K. and J.R. analyzed data; L.D.K. and P.D. wrote the paper.

This work was supported by fellowships from the Natural Sciences and Engineering Research Council of Canada and le Fonds de Recherche Québec-Santé to L.D.K., a studentship from the Groupe de Recherche sur le Systéme Nerveux Central (University of Montréal) to J.R., and the Canadian Institutes of Health Research and Natural Sciences and Engineering Research Council of Canada to L.S.-A. and P.D. We thank Shin-ichi Higashijima and Martin Meyer for sharing fish lines and reagents.

The authors declare no competing financial interests.

*L.D.K. and J.R. contributed equally to this work.

Correspondence should be addressed to Dr. Pierre Drapeau, Department of Neuroscience, Research Centre of the University of Montréal Hospital Centre, 900 rue Saint-Denis, Montréal, Quebec H2X 0A9, Canada. E-mail: p.drapeau@umontreal.ca.

DOI:10.1523/JNEUROSCI.1225-14.2014

Copyright $\odot 2014$ the authors $\quad 0270-6474 / 14 / 349644-12 \$ 15.00 / 0$
Our understanding of locomotor development has been greatly increased by studies in simple vertebrates because the network complexity is reduced to a smaller number of neuronal classes, yet the functional organization of spinal circuits remains conserved across vertebrates (Grillner and Jessell, 2009). The zebrafish (Danio rerio) is a valuable model in which to study motor development because of its amenability to genetic, pharmacological, and physiological analyses at embryonic stages with wellcharacterized immature behaviors (Saint-Amant, 2006).

The zebrafish embryo shows several motor behaviors. The earliest consists of transient slow, large amplitude repeating $1 \mathrm{~Hz}$ spontaneous coils of the trunk, occurring as early as $17 \mathrm{~h}$ postfertilization (hpf), soon after muscle innervation, but disappearing by $28 \mathrm{hpf}$ (Saint-Amant and Drapeau, 1998). Coiling behavior is driven not by chemical neurotransmission but by pacemaker ipsilateral caudal (IC) neurons in the rostral spinal cord that initiate regular periodic depolarizations in spinal neurons (including motoneurons) via gap-junction coupling, leading to an ipsilateral contraction of the trunk (Saint-Amant and Drapeau, 2000, 2001; Tong and McDearmid, 2012). By 21 hpf, embryos develop a glutamate-dependent response to touch consisting of 1-3 alternating contractions of the tail (Saint-Amant and Drapeau, 1998; Downes and Granato, 2006; Pietri et al., 2009). Neither the response to touch nor coiling depends on supraspinal inputs (Saint-Amant and Drapeau, 1998; Pietri et al., 2009). From 28 hpf onward, embryonic zebrafish show a swimming behavior driven 
by chemical glutamatergic and glycinergic neurotransmission and consisting of episodes of successive low-amplitude higher frequency $(10 \mathrm{~Hz})$ contractions in hatched $(52 \mathrm{hpf})$ larvae (Kimmel et al., 1995) that gradually increase to $40 \mathrm{~Hz}$ (Buss and Drapeau, 2001). Swimming activity recovers in the isolated spinal cord (Downes and Granato, 2006; Pietri et al., 2009) and can develop in the absence of descending inputs in the presence of NMDA (Chong and Drapeau, 2007), demonstrating that the neural circuitry within the spinal cord is sufficient to produce all of these earliest motor behaviors.

However, much remains to be understood about how early electrical coupling is integrated with and eventually dominated by chemical neurotransmission during network maturation. Here, we report the identification of a novel intermediate double coiling behavior that bridges the developmental gap between embryonic coiling and mature locomotion (swimming). Double coiling is dependent on both electrical and glutamatergic transmission and as such represents the output of a hybrid motor network.

\section{Materials and Methods}

Fish maintenance. Tübingen wild-type, touché heterozygous mutant (Low et al., 2010) and transgenic chx10:gal4 (Kimura et al., 2013) strains of adult zebrafish were maintained according to guidelines approved by the Animal Experimentation Ethics Committee, Universite de Montréal. Staging of embryos of, at this stage, undetermined sex, was performed as previously described (Kimmel et al., 1995).

Drug applications. All reagents were obtained from Sigma-Aldrich, unless otherwise noted. Embryos were anesthetized in $0.02 \%$ Tricaine and immobilized in $0.75 \%$ low-melting point agarose. A total of $3 \mathrm{~mm}$ CNQX, 5 mM APV, $1 \mu \mathrm{M}$ TTX, or saline was coinjected with $0.1 \%$ Fast Green dye and 2\% FITC cell-permeant fluorescent tracer dye, in Evans medium (134 mm NaCl, $2.9 \mathrm{~mm} \mathrm{KCl,} 2.1 \mathrm{~mm} \mathrm{CaCl}_{2}, 1.2 \mathrm{~mm} \mathrm{MgCl}_{2}, 10$ mm glucose, 10 mм HEPES, pH 7.8, with $\mathrm{NaOH}$ ) (Drapeau et al., 1999). Glass needles were pulled and mounted on a pipette holder, and $65 \mathrm{~ms}$ injections at 40 psi were delivered using a Picospritzer III (Parker Hannifin). Drugs were administered by bolus injections in the forebrain of the embryos (Knogler et al., 2010) as lesion studies have shown that this brain region is not involved in the production of embryonic motor behaviors (Saint-Amant and Drapeau, 1998). Drug- and sham-injected embryos were gently removed from agarose and allowed to recover for 30 min in Evans medium, before proceeding to video recording. In recordings with strychnine, the tip of the tail was removed and embryos were incubated in a solution of 0,5 , or $50 \mu \mathrm{M}$ strychnine in Evans for $1 \mathrm{~h}$ before recordings to allow for sufficient penetration of the drug without inducing the effects of chronic strychnine treatment on neural development (Downes and Granato, 2006; McDearmid and Drapeau, 2006; Côté and Drapeau, 2012).

Lesions. Full transections of the spinal cord were performed under a dissection microscope on anesthetized embryos with a fragment of a razor blade mounted to a plastic pipette tip in Evans medium containing $0.02 \%$ tricaine. Medium was replaced several times after the lesion, and animals were allowed to recover for $30 \mathrm{~min}$ before video recordings were taken.

Behavioral analysis. Embryonic motor activity in freely moving animals was recorded using a Point Gray Research Grasshopper and Flea2 digital video camera at 15-60 Hz, mounted on an Olympus dissection microscope (SZX7) fitted with epifluorescence. Embryos were kept at $28.5^{\circ} \mathrm{C}$ between recordings. Images were captured using PGR Flycap software and were then analyzed offline using ImageJ (National Institutes of Health) software, wherein $x, y$ coordinates and timing for each contraction were recorded using the Manual Tracker plugin.

Electrophysiology. Zebrafish embryos were dechorionated and anesthetized in $0.02 \%$ tricaine dissolved in Evans solution and dissected according to previously described procedures (Drapeau et al., 1999). Briefly, spinal neurons in somites 5-15 were selected for recording based on their soma size and position as visualized by oblique illumi- nation (Olympus BX61W1). To record spontaneous activity, $15 \mu \mathrm{M}$ D-tubocurarine (Sigma) was added to the Evans solution to block neuromuscular transmission. Electrophysiological recordings were done in the presence of 1-5 $\mu \mathrm{M}$ strychnine to block glycinergic events, or in $10 \mu \mathrm{M}$ CNQX to block glutamatergic events. Patch-clamp electrodes for spinal neuron recordings $(6-14 \mathrm{M} \Omega$ ) were pulled from borosilicate glass and were filled with the following intracellular solution (in $\mathrm{mM}$ ): 105 D-gluconic acid, $16 \mathrm{KCl}, 2 \mathrm{MgCl}_{2}, 10 \mathrm{HEPES}$, and $10 \mathrm{EGTA}$, adjusted to pH 7.2, 290 mOsm (Drapeau et al., 1999). Sulforhodamine B (0.1\%; Sigma) was also included in the patch solution to label the cells and confirm their identity after a recording. Standard whole-cell recordings from 23 to $30 \mathrm{hpf}$ larvae were obtained using an Axopatch 200B and a Molecular Devices CV 203BU headstage amplifier (Molecular Devices). Data were acquired at $40 \mathrm{kHz}$ and low-pass filtered at $10 \mathrm{kHz}$. Cells were held near their resting potential at $-65 \mathrm{mV}$ under voltage clamp unless otherwise specified. A maximum of three neural recordings were obtained from each embryo. Electrophysiological analyses were performed offline using Clampex 10.2 and Clampfit 10.2 software (Molecular Devices). The recordings were not analyzed if the resting potential was more positive than $-40 \mathrm{mV}$ or if the input resistance was $<500 \mathrm{M} \Omega$. After each recording, a series of fluorescent images of the rhodamine-filled cell and its axonal projections as well as bright-field images were collected with a QImaging camera (model 1394, QImaging) using Micro-Manager software (http://www.micro-manager.org/). Images were inverted and brightness/contrast was adjusted using Adobe Photoshop CS2 (Adobe Systems).

DNA microinjection and confocal microscopy. A plasmid containing five repeats of the upstream activating sequence driving synaptophysin-GFP and DsRed (Meyer and Smith, 2006) was injected at a concentration of 50 ng/ $\mu$ l into transgenic chx10:Gal4 zebrafish (Kimura et al., 2013) at the one-cell stage using a fine glass electrode and Picospritzer III (General Valve). Embryos were transferred to E3 ( $5 \mathrm{~mm} \mathrm{NaCl}, 0.17 \mathrm{~mm} \mathrm{KCL}, 0.33$ $\mathrm{mM} \mathrm{CaCl}_{2}, 0.33 \mathrm{~mm} \mathrm{MgSO}_{4}$ ) and raised as usual. Before imaging, embryos were dechorionated and anesthetized with $\mathrm{E} 3$ medium containing tricaine. Embryos were pinned on their side in a sylgard-coated dish with fine tungsten wire through the notochord.

Confocal imaging was performed on a Quorum WaveFX spinning disk system (Quorum Technologies) based on a modified Yokogawa CSU-10 head (Yokogawa Electric) mounted on an upright Olympus BX61W1 fluorescence microscope and equipped with a Hamamatsu ORCA-ER camera. Embryos were imaged using an Olympus LUM Plan 10× (NA 0.30 ) or $40 \times(\mathrm{NA} 0.80)$ water-dipping objective. A bright-field image and a set of stacked $z$-series images with $2 \times 2$ binning were collected sequentially in each channel Volocity software (Improvision). Step size for $z$-stacks ranged from 0.3 to $1 \mu \mathrm{m}$. $z$-stack maximum projections were created in ImageJ and combined to make color composites.

Statistical analyses. SPSS 21 (IBM) was used to assess data for statistical significance. All datasets were initially assessed for normality with the Shapiro-Wilk Test. For independent datasets with only two groups, we used the Students' $t$ test or Mann-Whitney $U$ test; and for data from multiple groups, we used a one- or two-way ANOVA with Bonferroni correction for post hoc testing $(p<0.05)$. In data obtained from the same group under different conditions, the paired-samples $t$ test or repeatedmeasures variation of ANOVA was used. $\chi^{2}$ test was used for nominal data. Error bars in bar graphs indicate the SE, and results are described as the mean \pm SE. Excel was used to create all graphs except the box plots, which were created online using the BoxPlotR application (http://boxplot.tyerslab.com/). In these plots, box limits indicate the 25th and 75th percentiles as determined by $\mathrm{R}$ software, center lines show the medians, whiskers extend 1.5 times the interquartile range from the 25 th and 75 th percentiles, and outliers are represented by open circles.

\section{Results}

\section{Transitional appearance of double coiling}

Zebrafish embryos show a developmental transition in motor behaviors. From 17 hpf onwards, they show repeating spontaneous contractions with a peak average frequency of one contrac- 
tion per second $(1 \mathrm{~Hz})$ at $19 \mathrm{hpf}$ that decreases to $<0.1 \mathrm{~Hz}$ by $26 \mathrm{hpf}$ and eventually disappears (Saint-Amant and Drapeau, 1998). These periodic spontaneous contractions consist of largeamplitude coils of the trunk during which the tip of the tail comes in contact with the head of the embryo (Fig. 1A) and at early stages tend to alternate sides. Beginning at $21 \mathrm{hpf}$, a coiling response is elicited by touch, whereas by $28 \mathrm{hpf}$ embryos will swim in response to touch or, to a lesser degree, spontaneously (Saint-Amant, 2006). In contrast to the slow frequency of coiling, when swimming is first observed in dechorionated embryos at $28 \mathrm{hpf}$, it consists of low-amplitude contractions occurring at a relatively high frequency of $10 \mathrm{~Hz}$. Transitions in spontaneous behavior between slow coiling and swimming have not been reported.

We examined spontaneous contractions at a high temporal resolution to probe for subtle changes during behavioral maturation. High-speed videos of spontaneous coiling events in dechorionated embryos partially immobilized in agarose revealed that spontaneous motor activity (coiling) could indeed be classified into at least two groups: single coils and double coils. Single coiling consisted of a single contraction of the trunk, after which the tail returned to its resting position (Fig. 1A). Double coiling, by contrast, consisted of two contralateral contractions of the trunk within $1 \mathrm{~s}$ (Fig. $1 B$ ). Between these two contractions, the tail did not return to a resting position, but instead the second contraction began rostrally before the first contraction had finished at the caudal end, forming a transient S-shape. These two types of coiling were also observable in nonimmobilized animals, and so we performed analyses of freely moving embryos to quantify the frequency of these events. These double coils first appeared at $24 \mathrm{hpf}, 7 \mathrm{~h}$ after the appearance of single slow coils and $3 \mathrm{~h}$ after the appearance of tactile responses, and with time increased in number relative to single coils, representing the majority of events by $27 \mathrm{hpf}$ (Fig. $1 C$; repeated-measures ANOVA shows significant effect of age with $p<0.01$ ). The peak frequency of double coiling, at $27 \mathrm{hpf}$, was $0.015 \pm 0.003 \mathrm{~Hz}$ (Fig. $1 C$, inset). A small number of events $(<5 \%)$ were also seen in these recordings that consisted of three or more alternating coils (data not shown).

We hypothesized that the relative increase in double coiling may introduce a mild refractory period in the motor network, thus contributing to the overall decrease in spontaneous activity over this period of time. When we measured the quiescent periods before and after each type of coil at $26 \mathrm{hpf}$, we found that on average the latency preceding a single or double coil was similar (Fig. $1 D ; 17.7 \pm 1.6$ and $20.2 \pm 2.8$ s, respectively; $p>0.20$ ),

B Double coil

E 성
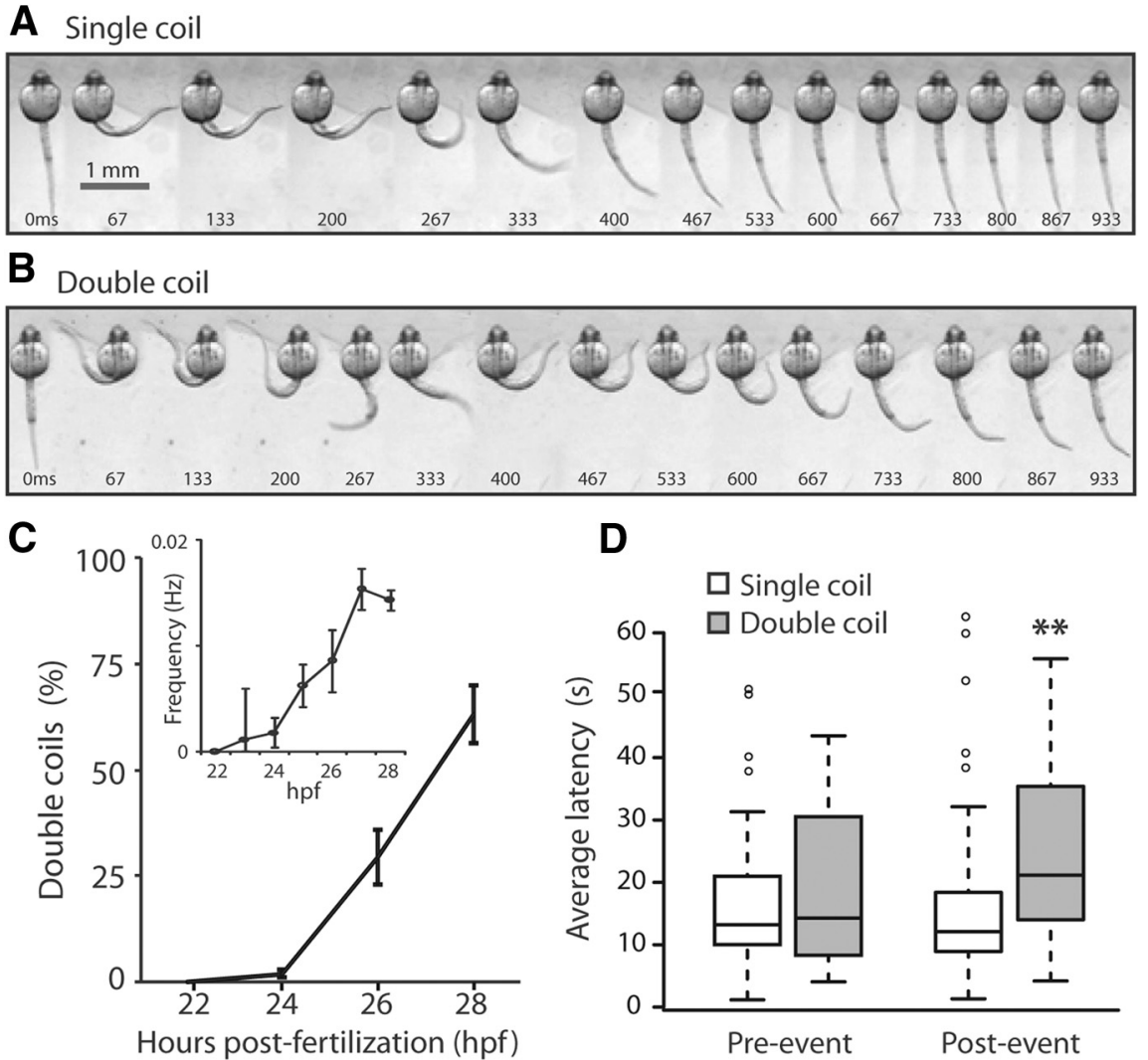

Pre-event Post-event

Figure 1. Double coiling appears during embryonic development and depresses network activity. $\boldsymbol{A}$, Still images taken at $67 \mathrm{~ms}$ intervals from a video recording of an agarose-embedded $26 \mathrm{hpf} \mathrm{embryo} \mathrm{exhibiting} \mathrm{a} \mathrm{spontaneous} \mathrm{single} \mathrm{coil} \mathrm{and} \mathrm{a} \mathrm{double} \mathrm{coil} \mathrm{(B).}$ Because of the embedding medium surrounding the head, the tail does not come into direct contact with the head in these images as it would in a nonimmobilized embryo. Scale bar: $\boldsymbol{A}$, same as for $\boldsymbol{B}$. $\boldsymbol{C}$, The relative percentage (\%) of double coils in freely moving bars indicate SE. $D$, Average latency, or time inactive, for a single coil (open bars) and a double coil (gray bars) in $26 \mathrm{hpf}$ embryos before (left, Preevent) and after (right, Postevent) $(N=30 ; p<0.01)$. Box plot showing the total event frequency $(\boldsymbol{E})$ and the percentage of double coils $(\boldsymbol{F})$ at $26 \mathrm{hpf}$ in touché mutants (dark gray bars) and wild-type siblings (white bars). $N_{\text {ctrl }}=12 ; N_{\text {touché }}$ $=12.0$ pen circles represent data outliers. See Materials and Methods for further details regarding box plot features.

whereas the latency after a double coil was nearly twice as long compared with a single coil (Fig. $1 D ; 14.8 \pm 1.1$ and $23.7 \pm 2.8 \mathrm{~s}$ for single and double coils, respectively; $p<0.005$ ), revealing that the presence of double coils did indeed depress the motor network for a short time interval. These results suggest that the appearance of double coils represents a discrete event in the stepwise maturation from a single coiling behavior to swimming.

\section{Double coiling does not depend on mechanosensory transduction}

The touch response first appears at $21 \mathrm{hpf}$ and consists of several alternating contractions of the tail beginning on the side contralateral to the stimulus (Saint-Amant and Drapeau, 1998; Pietri 
et al., 2009). One hypothesis for the appearance of double coils at a time shortly after the touch response is that double coils occur when a spontaneous single coil triggers a contralateral tactile response. We tested this hypothesis in a mutant line that is deficient in mechanosensation. Embryos of the touche mutant are completely unresponsive to touch as a result of defective sensory transduction (Low et al., 2010). In touché embryos, there should therefore be no possibility of self-triggering a touch reflex in response to spontaneous movement. High-speed videos of $26 \mathrm{hpf}$ embryos revealed no change in overall contraction frequency between touch-responsive controls and mutants touché siblings (Fig. $1 E ; 0.11 \pm 0.01$ and $0.094 \pm 0.01 \mathrm{~Hz}$, respectively; $p>0.20$ ). The percentage of double coiling was likewise not significantly different between control and mutant embryos (Fig. 1F; $30.5 \pm$ 4.7 and $27.4 \pm 8.2 \%$, respectively; $p>0.20$ ). Thus, double coiling was unaffected by a defect in mechanosensation, suggesting that it is a spontaneous behavior.

\section{Glutamatergic neurotransmission is required for double coiling}

Early spontaneous coiling in the embryonic zebrafish has been shown to rely on a central pattern generator with gap junctionmediated electrical synapses between spinal neurons and to be independent of chemical neurotransmission other than at the neuromuscular junction (Saint-Amant and Drapeau, 2000). To test the necessity of chemical neurotransmission for double coiling, we exposed embryos to the glutamate receptor antagonists CNQX or APV, which block AMPA or NMDA receptors, respectively. As previously reported (Saint-Amant and Drapeau, 2000; Pietri et al., 2009), the total event frequency was not significantly different between conditions (Fig. $2 A ; p>0.20$ ). However, CNQX treatment dramatically decreased the percentage of double coils throughout the period of 24-29 hpf (Fig. 2B, gray bars; e.g., at $26 \mathrm{hpf}$, percentage of double coils in controls was $29.3 \pm$ 6.5 vs $2.0 \pm 0.9 \%$ in CNQX treatment, $p<0.005$ from $25-29$ $\mathrm{hpf}$ ). APV had an intermediate effect and decreased double coiling to $<50 \%$ of control levels (Fig. $2 B$, black bars; e.g., at $26 \mathrm{hpf}$, double coiling frequency in APV treatment was $9.0 \pm 3.8 \%, p<$ 0.05 from 26-29 hpf). TTX treatment, which blocks voltagegated sodium channels, completely abolished motor activity, as previously described (data not shown) (Saint-Amant and Drapeau, 2001; Tong and McDearmid, 2012). These pharmacological results suggest that, although both single and double coils are mediated by electrical coupling, double coils are more akin to touch responses and swimming in their requirement of an additional component of glutamatergic neurotransmission.

\section{Normal frequency of occurrence of double coiling requires a descending excitatory drive from the hindbrain}

The results described above suggest that double coiling is a newly identified motor network output that can be clearly distinguished from both single coiling and the touch-evoked coiling response. To better understand how double coiling is generated, we probed for the minimal neural circuitry required to produce the behavior. It has been previously shown that the neural circuitry within the spinal cord is sufficient to produce single coiling and the touch response and therefore does not rely on descending input from the hindbrain (Downes and Granato, 2006; Pietri et al., 2009). The isolated $48 \mathrm{hpf}$ spinal cord is also able to generate swimming behavior if given enough time $(>20 \mathrm{~min})$ to recover from the transection (Downes and Granato, 2006) and swimming-related activity can develop in the absence of descending inputs in the presence of NMDA (Chong and Drapeau, 2007),
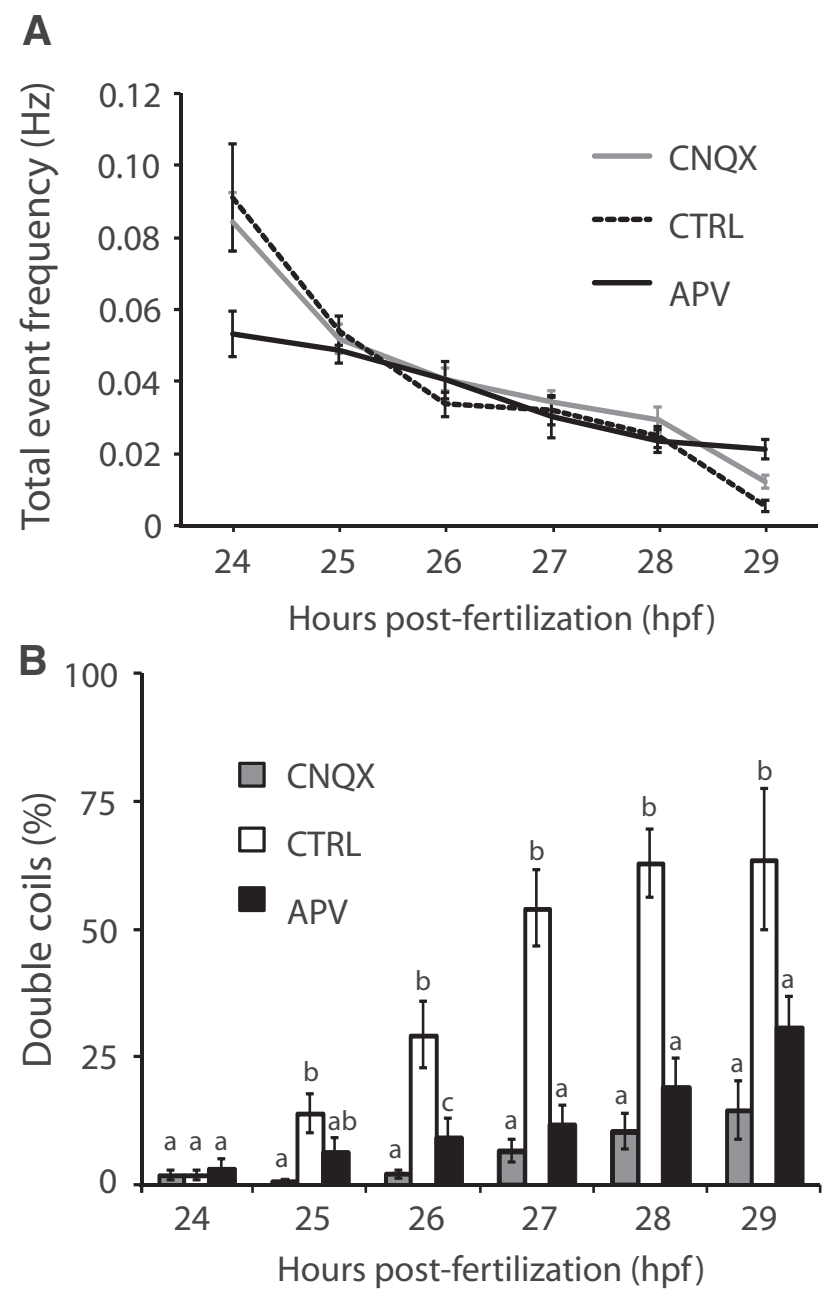

Figure 2. Glutamatergic neurotransmission is required for double coiling. $\boldsymbol{A}$, Overall event frequency is unaffected by glutamate receptor antagonists. $\boldsymbol{B}$, The relative proportion of double coils (\%) is significantly decreased after CNQX (gray bars) or APV treatment (black bars). Means that are not sharing a letter are significantly different at $p<0.05$. Error bars indicate $S E$. $N_{\text {control }}$ $=32 ; N_{\text {CNQX }}=43 ; N_{\text {APV }}=41$.

suggesting that the spinal cord is capable of producing the motor activity responsible for all of the earliest behaviors. Descending inputs from a small number of reticulopsinal neurons of the hindbrain have reached the rostral spinal cord by $20-24 \mathrm{hpf}$, and the second wave of supraspinal inputs does not arrive until 30-34 hpf (Mendelson, 1986).

To test the circuit requirements for double coiling, we lesioned 26 hpf embryos at the rostral end of the spinal cord, effectively removing all supraspinal structures, including the hindbrain (Fig. 3A). A touch response was still present following the lesion, confirming that the rostral spinal cord remained intact. As a control, embryos were lesioned at the level of the midbrain, leaving the hindbrain intact (Fig. $3 A$ ). The frequency of single coiling was similar between these two conditions $(0.11 \pm$ 0.02 vs $0.14 \pm 0.02 \mathrm{~Hz}$ for control vs lesioned embryos; $p>0.20$ ). The percentage of double coiling events was significantly reduced in embryos lacking supraspinal input compared with embryos receiving control lesions (Fig. $3 B ; 43.3 \pm 4.4$ vs $21.0 \pm 4.6 \%$ for control vs lesioned embryos; $p<0.005$ ), suggesting that the lesions resulted in a specific loss of double coiling. As a consequence, the overall contraction frequency decreased significantly in lesioned embryos $(0.30 \pm 0.03$ vs $0.20 \pm 0.02 \mathrm{~Hz}$ for control vs 

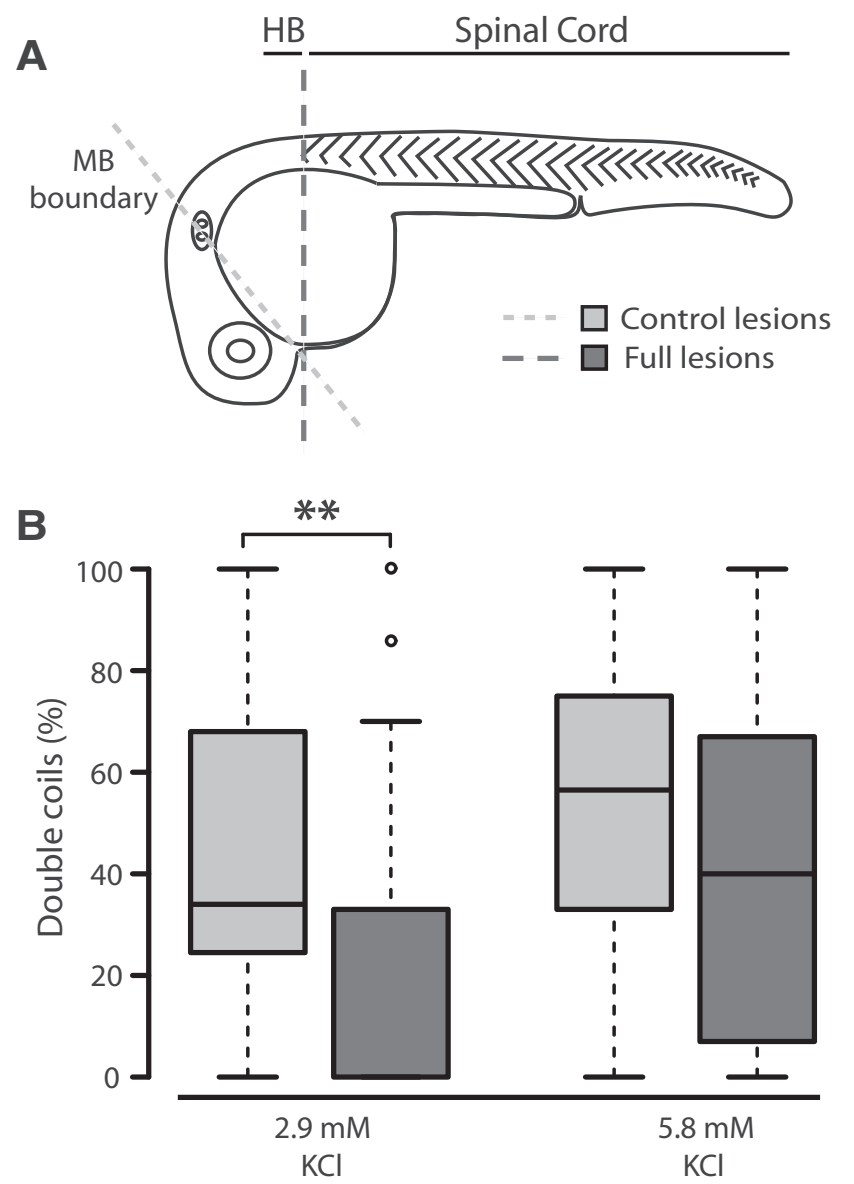

Figure 3. Double coils require a descending excitatory drive from the hindbrain. $A$, Diagram representing the site of control lesions at the midbrain (MB) boundary to leave the hindbrain (HB) intact, and full lesions at the caudal hindbrain to isolate the spinal cord. $B$, Box plot showing the proportion of double coils at $26 \mathrm{hpf}$ in control (gray bars) and fully lesioned embryos (dark bars) in normal $2.9 \mathrm{~mm} \mathrm{KCl}\left(\right.$ left, $N_{\text {ctrl }}=32, N_{\text {lesion }}=41$ ) and elevated $5.8 \mathrm{~mm} \mathrm{KCl}$ $\left(\right.$ right, $\left.N_{\text {ctrl }}=22, N_{\text {lesion }}=25\right) .{ }^{* *} p<0.01$.

lesioned embryos; $p<0.005)$. In addition, 22 of 41 lesioned embryos failed to produce any double coils compared with just 1 of 32 control embryos $(p<0.0001)$. Interestingly, the proportion of double coils in lesioned embryos was rescued to control levels when the concentration of $\mathrm{KCl}$ in the media was doubled to increase circuit activity (Fig. $3 B ; 54.1 \pm 6.8$ vs $42.5 \pm 6.8 \%$ for control vs lesioned embryos; $p>0.20$ ). Furthermore, in high $\mathrm{KCl}$ conditions, 6 of 25 lesioned embryos failed to produce double coils, compared with 3 of 22 control embryos ( $p>0.10$ ), consistent with rescue of the behavior. The average contraction frequency was also comparable in the high $\mathrm{KCl}$ condition $(0.18 \pm$ 0.02 vs $0.18 \pm 0.02 \mathrm{~Hz}$ for control vs lesioned embryos; $p>0.20$ ). These findings suggest that the spinal cord has the minimal circuitry capable of generating double coils but that the hindbrain may provide the necessary descending excitation to fully drive this behavior.

\section{Blockade of glycinergic neurotransmission promotes multiple coils}

Because of a high intracellular chloride gradient, glycine is depolarizing at embryonic stages and may excite spinal neurons by producing suprathreshold postsynaptic potentials but also shunt subsequent excitation, thereby raising the threshold for firing action potentials (for review, see Ben-Ari, 2002). Accumulation of glycine in the network in the zebrafish shocked mutant leads to a loss of spontaneous coiling activity and response to touch in the embryo (Cui et al., 2004, 2005), suggesting that the regulation of glycinergic signaling is important in the production of early behaviors. Previous studies using strychnine, a glycine receptor antagonist that disrupts glycinergic signaling in the zebrafish embryo, have shown dose-dependent effects on motor behaviors. Low doses of strychnine $(1 \mu \mathrm{M})$ do not appear to alter the average frequency of spontaneous coiling (Saint-Amant and Drapeau, $2000)$, whereas high doses $(\geq 400 \mu \mathrm{M})$ significantly decrease spontaneous coiling frequency and can also disrupt the touchevoked response and swimming (Downes and Granato, 2006). However, high concentrations of strychnine may lead to offtarget effects, such as by blocking nicotinic acetylcholine receptors in muscles and neurons (García-Colunga and Miledi, 1999). We therefore chose to examine the role that glycine neurotransmission might play in double coiling using moderate doses of strychnine.

Embryos were bathed for $1 \mathrm{~h}$ in embryo medium containing 0, 5 , or $50 \mu \mathrm{M}$ strychnine to allow for penetration of the drug, and spontaneous coiling activity was subsequently analyzed at 24, 26, and $28 \mathrm{hpf}$. In the absence of strychnine, multiple $(3+)$ coils were rare and accounted for $<2 \%$ of all events in control embryos (Fig. $4 A-C)$. At all three ages examined, however, strychnine had a dose-dependent effect of increasing the proportion of multiple coils and correspondingly decreased the proportion of single coils (Fig. $4 A-C$ ). This result is particularly interesting considering that the blockade of glutamatergic signaling resulted in a large increase in the proportion of single coils at the expense of a loss of double coiling (Fig. 2B), the opposite of the outcome seen here. An age-dependent effect of strychnine was also seen, as $50 \mu \mathrm{M}$ strychnine treatment only slightly increased the proportion of multiple coils in $24 \mathrm{hpf}$ embryos (to $<5 \%$ of all events), whereas by 26 and $28 \mathrm{hpf}$ the proportion of multiple-coil events was increased to $15 \%-20 \%$ of all events, a much larger increase over control values (Fig. $4 A-C$ ).

Coiling events with three or more contractions had a tendency to be followed by long periods of inactivity. Data pooled across strychnine-treated $26 \mathrm{hpf}$ embryos show that multiple-coil events with three or more contractions were on average followed by a latency more than three times longer than the latency to the next event following a single-coil in control embryos (Fig. 4D; $p<$ 0.001 ). Interestingly, the latencies following both single and double coils were also significantly increased in embryos treated with strychnine compared with single-event latencies in controls, but to a lesser extent than seen with multiple coil events (Fig. $4 D ; p<$ 0.05 and $p<0.005$ for single and double coiling, respectively). This is likely the result an overall reduction in event frequency related to the depressive effect of the multiple coiling on the network.

These results suggest that, despite the fact that glycine is depolarizing at this developmental stage (Saint-Amant and Drapeau, 2000), glycinergic neurotransmission itself does not appear to drive double coiling. Instead, the role of glycinergic inputs during spontaneous coiling events may be to limit the contralateral activation of the spinal cord by shunting the membrane resistance and keeping the membrane potential below action potential threshold.

\section{Double coiling-related activity recorded in primary motoneurons}

To examine the physiological characteristics of double coiling at the cellular level, we recorded from identifiable primary motoneurons (MNs) of the spinal cord between 24 and 30 hpf. Cur- 

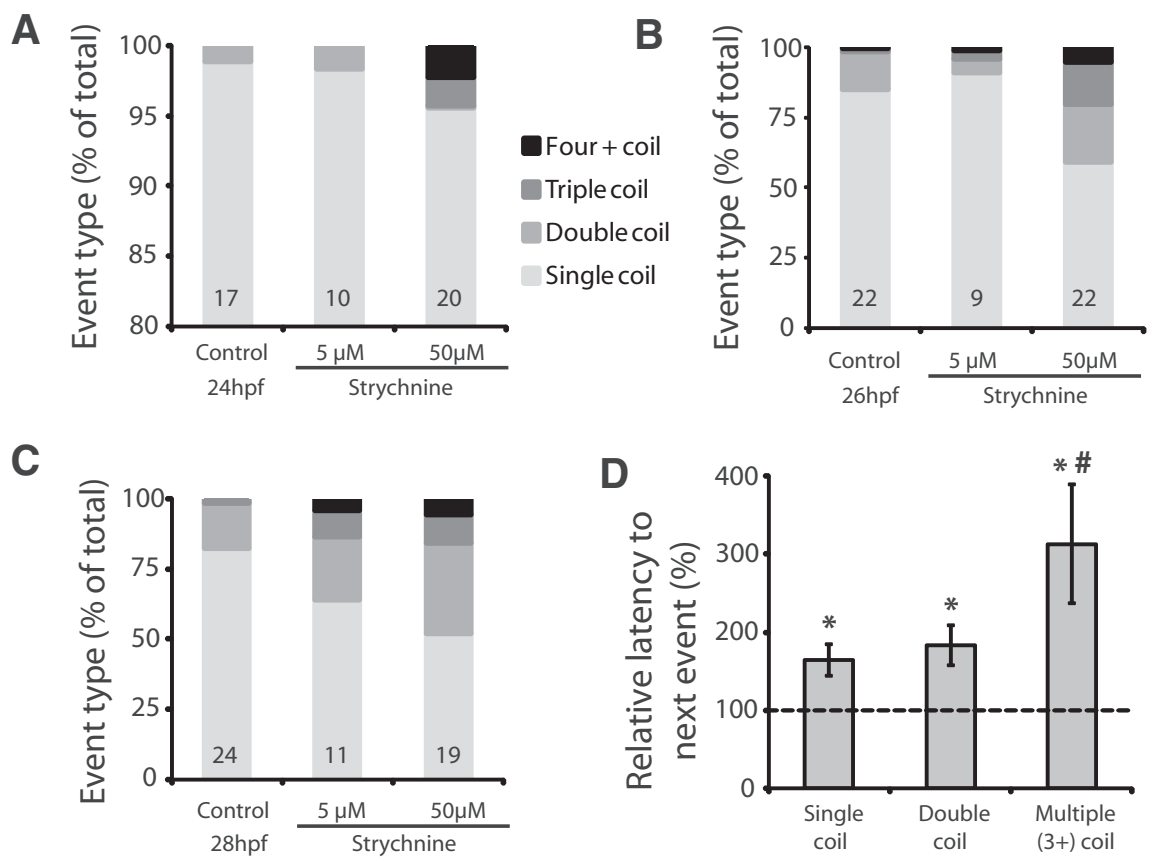

Figure 4. Decreased glycinergic signaling results in an increase in multiple coiling. Relative percentage of spontaneous events showing single, double, triple, and four and more $(4+)$ coils in embryos treated with strychnine and untreated controls at $(\boldsymbol{A}) 24$ hpf, (B) $26 \mathrm{hpf}$, and (C) $28 \mathrm{hpf}$. Note the expanded $y$-axis scale in $\boldsymbol{A}$. Numbers at the base refer to the numbers of embryos in each condition. $\boldsymbol{D}$, Average latencies in strychnine-treated $28 \mathrm{hpf}$ embryos after a single, double, or multiple $(3+)$ coil as normalized to single-coil latency in control embryos (dashed line). $N_{\text {ctrl }}=22 ; N_{\text {strychnine }}=31 .{ }^{*} p<0.05$ compared with control. ${ }^{\#} p<0.05$ within strychnine-treated embryos.

rent injections were always capable of inducing burst firing of action potentials in the $\mathrm{MN}$ that lasted the duration of the current pulse (Fig. 5A). Whole-cell voltage-clamp recordings in MNs showed a regular pattern of gap junction-driven periodic inward currents (PICs) (Fig. $5 B, C, E$ ) that underlie single coiling, as previously described (Saint-Amant and Drapeau, 2000). Also of note was the presence of distinct fast large peaks ( $\geq 15 \mathrm{pA})$ increasing in amplitude and frequency with age (compare Fig. $5 C$, black arrowheads, with Fig. 5D). These peaks were determined to be synaptic glutamatergic events following several observations. Unlike depolarizations resulting from electrical coupling, the fast peaks reversed at membrane potentials more positive than the cation reversal potential $(\sim 0 \mathrm{mV}$; Fig. $5 D)$. Pharmacological experiments showed that these fast peaks were not blocked by strychnine $(N=$ 9 of 9; Fig. 5D) but disappeared upon addition of $10 \mu \mathrm{M} C N Q X$ to the extracellular solution to block AMPA receptors $(N=5$ of 5 ; Fig. 5E). The largest glutamatergic peaks coincided with the electrical inward currents and had amplitudes up to $300 \mathrm{pA}$, although much smaller glutamatergic peaks resembling miniature EPSCs with amplitudes of 5-10 pA were also seen in the interval between events (Fig. 5E, gray arrowheads and inset). These small putative glutamatergic mEPSCs were also abolished by CNQX application.

In addition to electrical and glutamatergic currents, synaptic bursts (SBs) appearing as inward currents and corresponding to glycine-mediated chloride extrusion (Saint-Amant and Drapeau, 2000) were present at a similar frequency and duration as the periodic electrical currents (Fig. 5B). Glycinergic synaptic bursts onto $\mathrm{MNs}$ are thought to originate from commissural glycinergic interneurons, and paired current-clamp recordings have shown that these glycinergic inputs arrive at a $\mathrm{MN}$ at the same time that a gap junction-driven inward current is depolarizing the contralateral MN (and its electrically coupled neighbors) and driving a single coil on that side of the trunk (Saint-Amant and Drapeau,
2001). In agreement with previous studies (Saint-Amant and Drapeau, 2000, 2001), synaptic bursts were completely absent in recordings where the extracellular solution contained $5 \mu \mathrm{M}$ strychnine to block glycine receptors $(n=11$ of 11$)$. Under current clamp, both glycinergic postsynaptic potentials as well as gap junctiondriven depolarizations were capable of driving action potentials in motoneurons in embryos $\geq 25 \mathrm{hpf}$ (Fig. $5 G$ ), although to a far greater degree with electrical than glycinergic inputs.

Given our knowledge that gap junction-driven depolarizations of MNs drive single coiling (Saint-Amant and Drapeau, 2000), we therefore hypothesized that a double coil would be distinguishable from a single coil by the close succession $(<1 \mathrm{~s})$ of a mixed event composed of electrical then glycinergic inputs in a $\mathrm{MN}$, in either order, which would correlate to the closely timed depolarization of an ipsilateral then contralateral $\mathrm{MN}$ and therefore an alternating double coil. It is important to note that the glycinergic synaptic burst itself is a correlate of contralateral electrical depolarization and is not thought to drive the coiling activity (Saint-Amant and Drapeau, 2001). Indeed, electrophysiological recordings from MNs clearly showed mixed electrical/glycinergic events (Fig. $5 \mathrm{~B}, \mathrm{H}, \mathrm{I}$ ) that we interpret as physiological correlates of double coiling. These mixed events were first observable in a minority of MN recordings from 23 to $24 \mathrm{hpf}$ embryos but were present in all MN recordings from embryos aged 26-30 hpf, correlating well with the increasing proportion of double coils observed in the intact embryo at these ages (Fig. 2B) and thus providing a useful readout of fictive double coiling. Evidence of fictive multiple coils ( $\geq 3$ coils) was also seen under conditions where strychnine was present and synaptic bursts were no longer present (Fig. $5 J$ ). These results mimic our behavioral results in strychnine (Fig. 4) and suggest that glycine (and synaptic bursts) do not drive coiling behavior and indeed may act to limit excitation in the network under physiological conditions.

\section{Blockade of glutamatergic neurotransmission abolishes mixed events in motoneurons}

To confirm the requirement of glutamatergic neurotransmission for the mixed events that represent fictive double coils, we perfused CNQX into the bath and looked for an effect on MN activity patterns. Bath application of $10 \mu \mathrm{M}$ CNQX blocked all glutamatergic synaptic events in our recordings and was sufficient to eliminate all mixed events without affecting the overall frequency of electrical or glycinergic synaptic burst events alone (Fig. $6 A ; N=4$ ) or the amplitudes of these currents (Fig. $6 B ; p>0.20$ for both PICs and SBs). The change in amplitudes of mixed events could not be analyzed because they were absent or extremely rare in the presence of CNQX. Subsequent washing out of CNQX showed that the mixed events recovered, confirming that their loss was not the result of an artifact of the long recording (Fig. 6C,D). These results corroborate our behavioral findings and suggest that the mixed events recorded in MNs are physiological corre- 
A
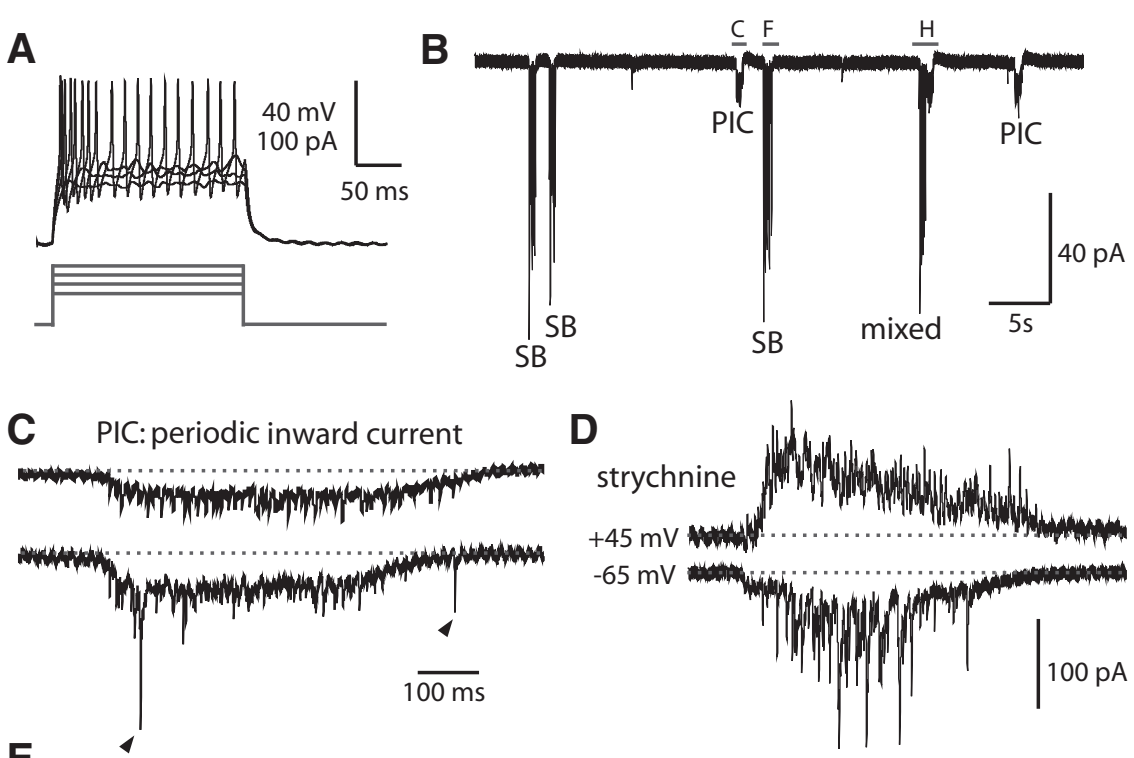

E

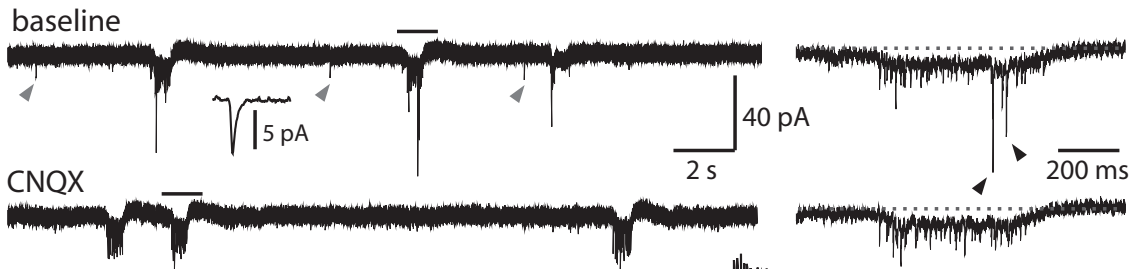

F SB:synaptic burst

G
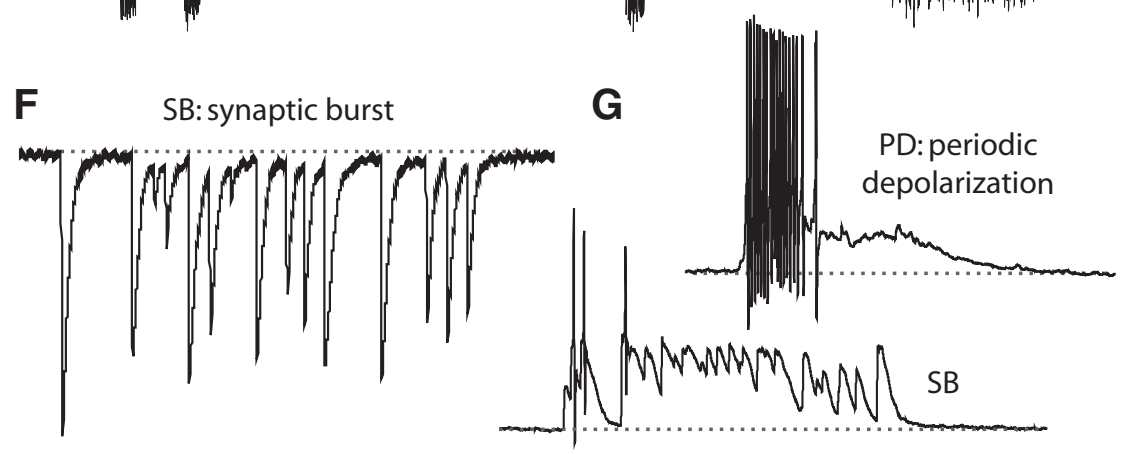

H
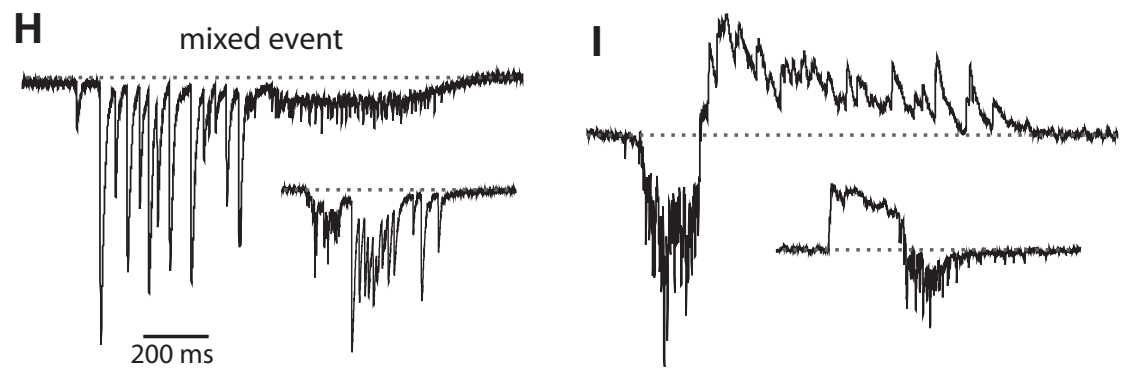

$\mathbf{J}$

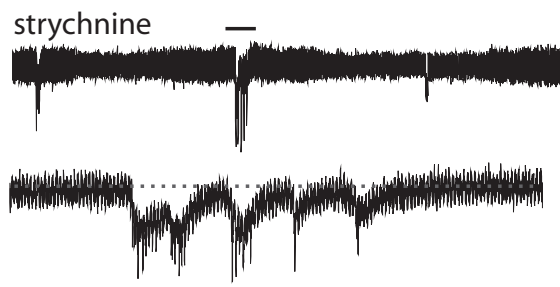

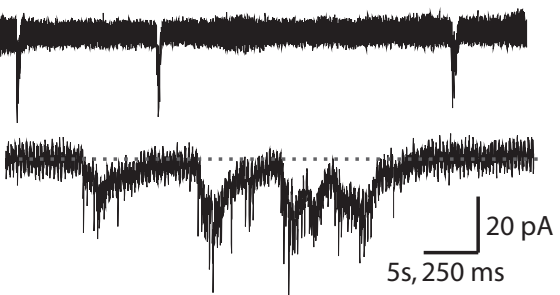

Figure 5. Motoneurons show mixed electrical and synaptic events corresponding to glutamate-dependent fictive double coils. $A$, Representative current injections showing burst firing of action potentials in embryonic MNs. Top, Current-clamp recording from neurons. Bottom, Current steps. B, Patterned activity in a 25 hpf embryo includes gap junction-driven PICs, SBs, and mixed events. lates of fictive double coiling behavior and that glutamatergic signaling is indeed necessary for double coiling.

\section{Candidate glutamatergic interneurons in the early motor network}

Having described novel glutamate-dependent double coiling by behavioral, pharmacological, electrophysiological, genetic, and lesion analyses, we next sought to identify the key interneurons in the neural network underlying this motor activity. There are a

\section{$\leftarrow$}

Holding potential in this trace and all others is $-65 \mathrm{mV}$ (unless otherwise indicated). Events in the indicated region are expanded in panels $\boldsymbol{C}, \boldsymbol{F}$, and $\boldsymbol{H}$. $\boldsymbol{C}, \mathrm{PIC}$ are low-amplitude inward currents produced by the electrical coupling of neurons that often coincide with synaptic glutamatergic events. Top, PIC (from region indicated in $\boldsymbol{B}$ ) that shows no glutamatergic peaks. Bottom, PIC in the same MN that does show synaptic glutamatergic events (arrowheads). Baseline is shown as a dotted gray line for reference in this and subsequent figures. Vertical scale same as for $\boldsymbol{B}$, but note different time scale. $\boldsymbol{D}$, Examples of PICs under voltage clamp seen in an older embryo (27 hpf) with glutamatergic events of increased amplitude and frequency. These recordings were done in the presence of $5 \mu \mathrm{m}$ strychnine. Some of the currents are reversed at a positive holding potential of $45 \mathrm{mV}$ (top) compared with a normal holding potential ( $-65 \mathrm{mV}$, bottom), corresponding to purely glutamatergic events. Time scale same as in $\boldsymbol{C}$, but note different vertical scale. $\boldsymbol{E}$, Excerpt from a voltage-clamp recording in a $26 \mathrm{hpf}$ embryo showing three examples of PICs at baseline (left, top) and in the presence of $10 \mu \mathrm{M}$ CNQX (left, bottom). Small amplitude peaks occurring between events resembling glutamatergic $m E P S C s$ are highlighted with gray arrowheads, and an average of several peaks $(n=19)$ is shown in the inset at 100 times expanded time scale. Regions indicated with a black bar are shown on a 10 times expanded time scale at the right for each condition. Glutamatergic peaks occurring during a PIC are indicated with black arrowheads. $\boldsymbol{F}$, Synaptic bursts (from region indicated in $\boldsymbol{B}$ ) are strychnine-sensitive glycinergic currents of large amplitude with a similar duration to PICs. $\mathbf{G}$, Representative whole-cell current-clamp recordings from a primary MN in a $26 \mathrm{hpf}$ embryo. Resting membrane potential of the neuron is $-60 \mathrm{mV}$, scale same as in C. Top, Spontaneous periodic depolarization (PD) triggers burst firing of action potentials. Bottom, Glycinergic SB can also trigger action potentials. $\boldsymbol{H}$, Representative whole-cell voltage-clamp recordings of a mixed events (top trace from region indicated in $\boldsymbol{B}$ ) during which a PIC (with or without glutamatergic peaks) and an SB occur in close (subsecond) succession in either order. Vertical scale for uppermost trace same as in $\boldsymbol{B}$, but note different time scale. Inset, Scaled down by $50 \%$. I, Examples of mixed events from the same $M N$ as in $\mathbf{G}$ under voltage clamp at an intermediate holding potential of $-20 \mathrm{mV}$ (more positive than the chloride reversal potential of $\sim-35 \mathrm{mV}$ ) showing that glycinergic currents, but not electrical and glutamatergic currents, are reversed. Scale for top trace and inset same as for $\boldsymbol{H}$. $J$, Top, A 100 s excerpt of an MN voltage-clamp recording in a $26 \mathrm{hpf}$ embryo in the presence of $5 \mu \mathrm{m}$ strychnine showing that glycinergic synaptic bursts are absent, but gap junction-driven PICs remain. Bottom, left, An expanded view of the region indicated in the top trace showing an event with many individual electrical PICs occurring closely together in time and resembling a fictive multiple coil. Bottom, right, Example from a different MN. 


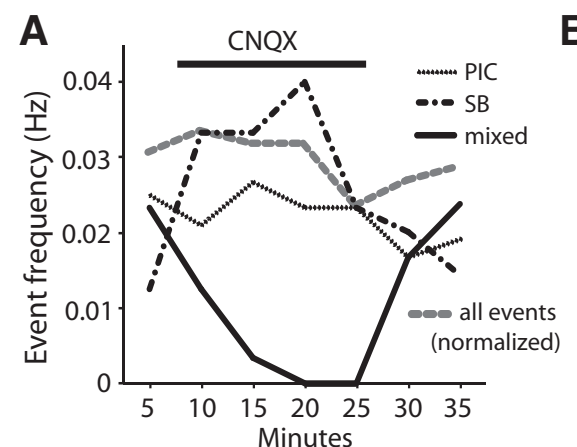

C

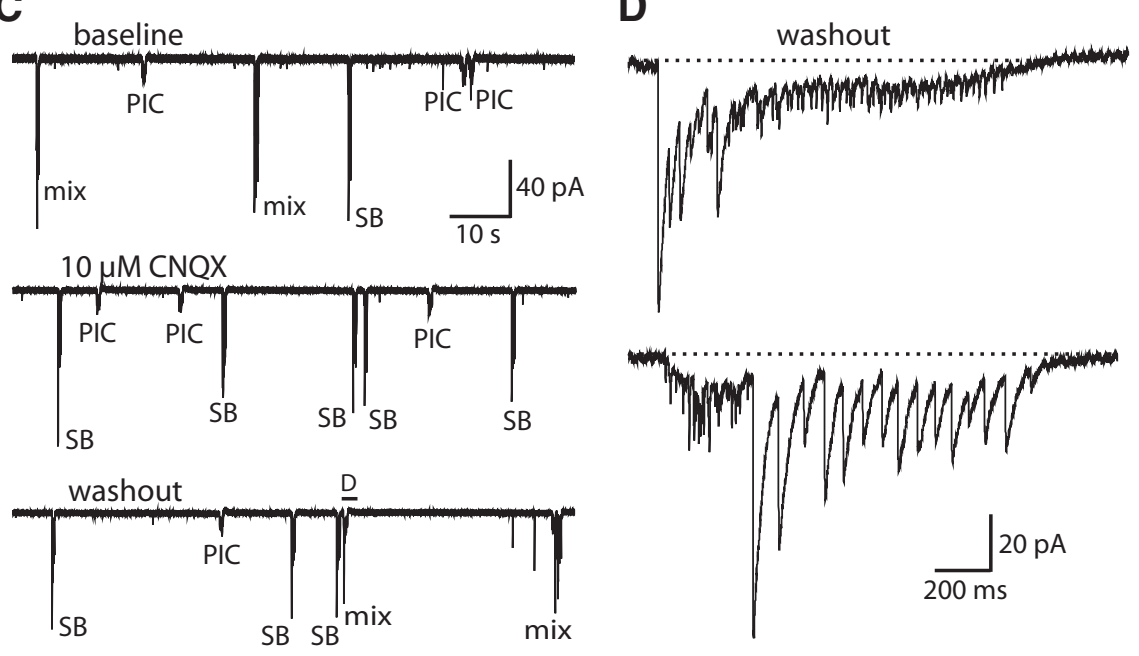

Figure 6. Blockade of glutamatergic neurotransmission abolishes mixed events in motoneurons but not SBs or PICs. A, Average frequency of each type of event as well as the total (normalized) event frequency during a representative whole-cell recording $(N=4)$ in which $10 \mu \mathrm{M}$ CNQX was washed into and out of the extracellular recording solution. $\boldsymbol{B}$, The average maximum amplitudes of SBs and mixed events (left) and PICS (right) at baseline are not significantly changed by the presence of CNQX. Amplitude of mixed events is not given for the CNQX condition because these events were rare or absent. Maximum amplitude of $\mathrm{PICs}$ at baseline ignored glutamatergic peaks. C, Excerpts of voltage-clamp recordings from the experiment in $\boldsymbol{A}$ showing the presence of mixed events at baseline (top), the selective loss of mixed events after the addition of CNQX (middle), and their subsequent recovery after CNQX washout (bottom). D, Representative mixed events after CNQX washout. Top, From the region indicated in the washout recording in C. Bottom, From the same MN.

limited number of classes of interneurons in each somite of the spinal cord at this age, and these interneurons have been well characterized in terms of dorsoventral soma position, axonal morphology, and neurotransmitter phenotype (Bernhardt et al., 1990; Hale et al., 2001; Higashijima et al., 2004). Electrophysiological recordings in the early embryo have shown that, in addition to the primary MNs, at least four classes of spinal interneurons are active at $24 \mathrm{hpf}$ : ipsilateral caudally projecting (IC), ventral lateral descending (VeLD), circumferential descending (CiD), and commissural primary ascending (CoPA) interneurons (Saint-Amant and Drapeau, 2001). IC cells have pacemaker properties and are thought to initiate spontaneous network activity in the embryonic spinal cord via electrical coupling, suggesting that at early stages these neurons do not connect to the network with chemical synapses (Tong and McDearmid, 2012). VeLDs are GABAergic (Batista et al., 2008), whereas both CoPA and CiD neurons are glutamatergic (Higashijima et al., 2004; Kimura et al., 2006; Batista et al., 2008). Therefore, the CoPA and CiD neurons are the most likely candidates to mediate the glutamatergic neurotransmission important for double coiling behavior at early stages and we recorded from each type.

By $26 \mathrm{hpf}$, CoPA axons can span up to 12 somites (Bernhardt et al., 1990), suggesting that these neurons have developed exten- sive axonal projections by the onset of double coiling behavior. In embryos as young as $24 \mathrm{hpf}$, filled CoPAs had axons that crossed the midline and ascended dorsally and rostrally for several somites (Fig. 7B). CoPA neurons receive direct glutamatergic input from sensory RohonBeard (RB) cells and relay this excitation during evoked behaviors (Pietri et al., 2009). We therefore recorded from these cells in 24-29 hpf embryos to examine how their activity might contribute to double coiling. The only spontaneous activity seen in CoPAs was a long duration $(>1 \mathrm{~s}), \sim 20 \mathrm{mV}$ amplitude depolarization that was often sufficient to elicit a single action potential at the onset of the depolarizing plateau (Fig. $7 A ; N=20$ ). In the absence of sustained firing, these recordings suggest that CoPAs are not as important for spontaneous behaviors as they are for the touch response in their proposed role as a sensory interneuron (Pietri et al., 2009).

\section{Glutamatergic $\mathrm{CiD}$ interneurons are} active during spontaneous behaviors $\mathrm{CiD}$ neurons express the transcription factor chx10 and are part of the V2a population of excitatory premotor interneurons in zebrafish as well as in other vertebrates (Kimura et al., 2006). Many $\mathrm{CiD}$ soma are contacted by the ascending axons of the CoPA sensory interneurons in the dorsal longitudinal fasciculus and may thus form part of a common path to relay excitation toward the contralateral MN during evoked responses (Pietri et al., 2009). We recorded from CiD neurons in 24-30 hpf embryos to examine their intrinsic and synaptic properties during the period of spontaneous double coiling.

At $24 \mathrm{hpf}$, CiD neurons had ipsilateral axons projecting ventrally and caudally that were varied in length from one to several somites (Fig. 7C). Whole-cell current-clamp recordings showed that $\mathrm{CiDs}$ responded to current injections with a maximum of 1-3 action potentials at earlier ages, but became capable of burst firing with large undershoots by $27 \mathrm{hpf}$ onward (Fig. 7D). CiDs were spontaneously very active, and there was clear evidence of electrical coupling to the network at all ages studied. Like MNs, CiDs receive gap junction-driven periodic inward currents that by 26-27 hpf were sufficient to produce bursts of action potentials under current-clamp conditions (Fig. 7E, top). Glutamatergic synaptic events could also be seen on top of the electrical currents (Fig. $7 F$ ) and became increasingly apparent in size and frequency with age. Additionally, CiDs received depolarizing glycinergic synaptic bursts that led to action potential firing by 27 hpf (Fig. 7E, bottom). Finally, voltage-clamp recordings revealed mixed events in CiDs (Fig. $7 G$ ) that were very similar to those seen in MN recordings (Fig. $5 \mathrm{H}$ ), suggesting that the activity of the CiD is very similar to that of the MN.

Paired current-clamp recordings of CiD cells and ipsilateral MNs in embryonic zebrafish spinal cord have shown that their 
gap junction-driven depolarizations are synchronous, suggesting that they are part of a local coordinated group of ipsilateral neurons that are electrically coupled (Saint-Amant and Drapeau, 2001). Simultaneous recording of a $\mathrm{CiD}$ in cellattached mode and an ipsilateral $\mathrm{MN}$ under whole-cell voltage-clamp located two somites caudally showed that the CiD fired several action potentials during the electrical component and before the onset of but not during the glycinergic component in a mixed event in the MN (Fig. $7 H$ ). Furthermore, the CiD received glycinergic inputs in the form of a synaptic burst in synchrony with the MN (Fig. 7I), suggesting that, in addition to being electrically coupled, these cells receive coordinated synaptic input from the same or similar unidentified contralateral glycinergic neurons.

These results show that the intrinsic and synaptic properties of $\mathrm{CiD}$ neurons are maturing at the time double coiling first appears. CiDs are spontaneously highly active, capable of firing several action potentials and show activity that is very similar to the mixed events seen in MNs that we propose underlie double coils. These data suggest that the CiD plays an important role in propagating ipsilateral excitation in the early embryo via electrical and/or chemical neurotransmission.

\section{CiD neurons contact ipsilateral MNs and other CiDs with putative glutamatergic synapses}

The activity pattern of the CiD neuron suggested that it may provide important ipsilateral drive; therefore, to investigate the development of synaptic properties of these cells for glutamatergic neurotransmission, we used the Gal4/upstream activating sequence system to drive mosaic expression of synaptophysin-GFP and DsRed (syp:GFP-DsR) (Meyer and Smith, 2006) in chx10:Gal4 transgenic fish (Kimura et al., 2013). Confocal imaging revealed that the majority of DsRedlabeled cells had a CiD morphology, as identified by a caudal axon projecting first ventrally, and then proceeding dorsally and laterally. It has been previously reported that CiDs are found in the spinal cord by $22-23 \mathrm{hpf}$ and begin to extend their axons shortly thereafter (Bernhardt et al., 1990). Before $24 \mathrm{hpf}$, cells expressing DsRed were clearly seen in the spinal cord, but their axons were very short and cell identity was ambiguous. By $24 \mathrm{hpf}$, coinciding with the onset of double coils, CiDs were clearly seen with axons spanning up to two caudal somites (Fig. 8A). Except for
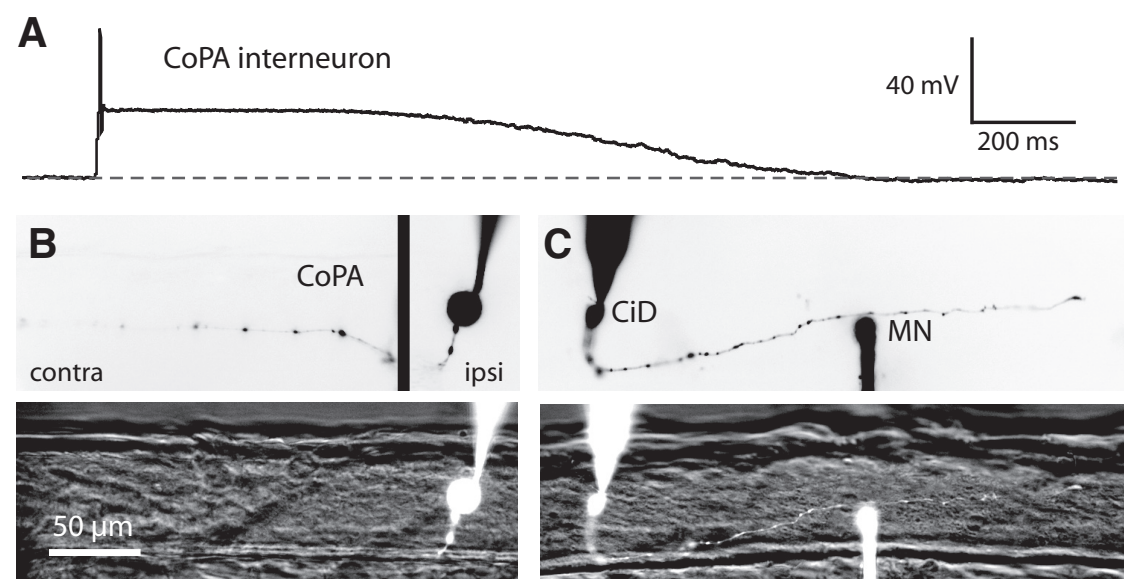

D CiD interneuron

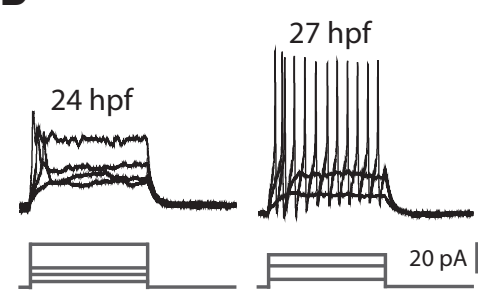

E

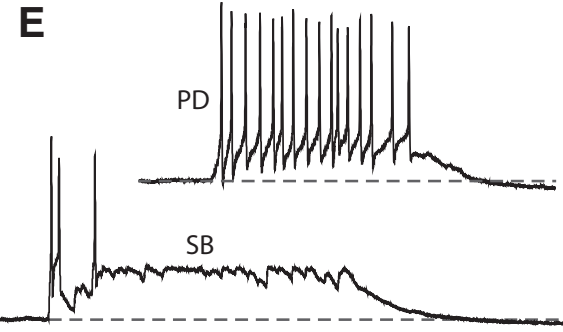

F
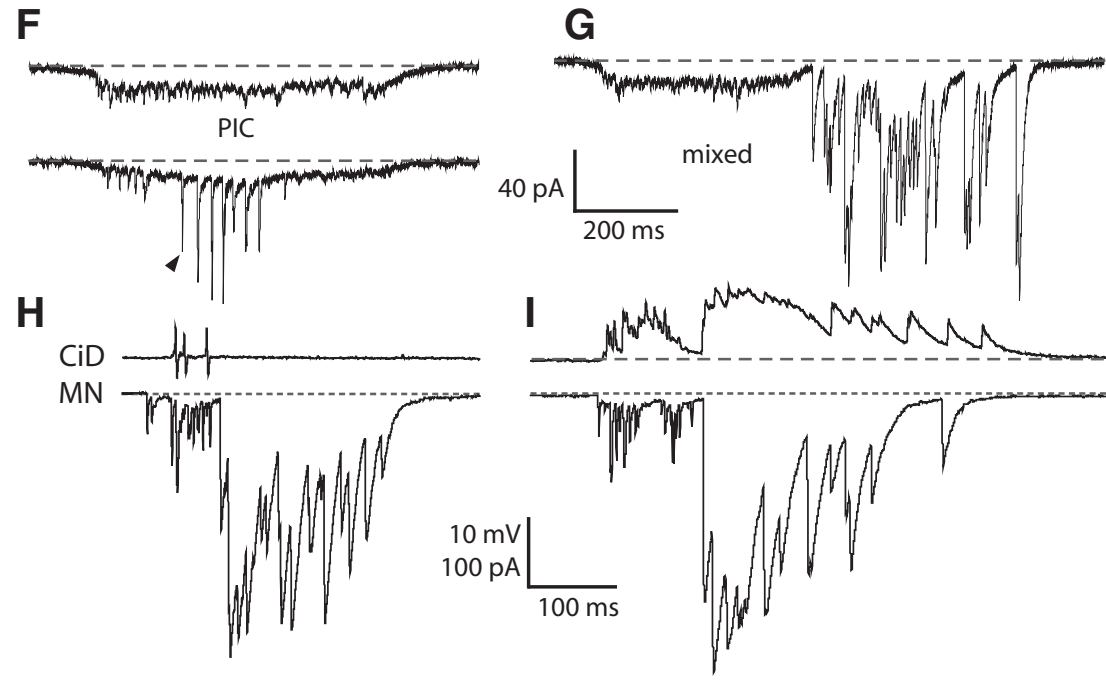

Figure 7. Glutamatergic CiD interneurons are highly active at early embryonic stages and, in contrast to COPAs, fire bursts of action potentials during spontaneous behaviors. $A$, Representative whole-cell current-clamp recording from a CoPA neuron showing a long duration depolarizing plateau event that often triggers an action potential at the onset. Resting membrane potential of the neuron is $-60 \mathrm{mV}$. Baseline is shown as a dashed gray line for reference in all traces. $\boldsymbol{B}$, Example of a filled CoPA after a whole-cell recording. Top, Inverted fluorescent image of the rhodamine-filled ipsilateral cell body (right, ipsi) merged with the filled ascending contralateral axon (left, contra). Bottom, Filled CoPA (ipsilateral plane only) in fluorescence against the spinal cord in bright-field. Rostral is to the left and dorsal is to the top (the same for all subsequent figures). C, Example of filled neurons from a simultaneous whole-cell recording of a CiD and ipsilateral MN. Top, Inverted fluorescent image of rhodamine-filled cells and the descending ipsilateral $\mathrm{CiD}$ axon (the ventral MN axon is obscured by the recording electrode). Bottom, Filled cell bodies in fluorescence against the spinal cord in bright-field. Scale bar, same as in $\boldsymbol{B}$. $\boldsymbol{D}$, Representative current injections showing single versus burst firing of action potentials in CiD neurons at $24 \mathrm{hpf}$ (left) and $27 \mathrm{hpf}$ (right), respectively. In each set, top traces are currentclamp recording from neurons and bottom traces are current steps. Scale bar, top traces, same as in $\boldsymbol{A}$. $\boldsymbol{E}$, Representative whole-cell current-clamp recordings from a CiD neuron. Resting membrane potential of the neuron is $-60 \mathrm{mV}$. Scale, same as in $A$. Top, Spontaneous periodic depolarization (PD) triggers burst firing of action potentials in a $27 \mathrm{hpf} \mathrm{embry0.} \mathrm{Bottom,} \mathrm{Glycinergic} \mathrm{SB} \mathrm{can}$ also trigger action potentials. $F$, Examples of PICs seen under voltage clamp in a CiD. Top, PIC that shows no glutamatergic peaks. Bottom, PIC from the same CiD that does show synaptic glutamatergic events (arrowhead indicates the first of several). Holding potential is $-65 \mathrm{mV}$; scale shown in $\mathbf{G} . \mathbf{G}$, Representative example of a mixed event seen under voltage clamp in a CiD. $\boldsymbol{H}$, Simultaneous recordings from a $28 \mathrm{hpf} \mathrm{embryo} \mathrm{of} \mathrm{a} \mathrm{CiD} \mathrm{(cell-attached} \mathrm{mode,} \mathrm{top)} \mathrm{and} \mathrm{an} \mathrm{MN} \mathrm{(voltage} \mathrm{clamp,} \mathrm{bottom)} \mathrm{during} \mathrm{fictive}$ double coiling. $\boldsymbol{I}$, Whole-cell voltage-clamp recording from the same pair of neurons as in $\boldsymbol{H}$ to show the similar patterning of activity during fictive double coiling. Holding potential is $20 \mathrm{mV}$ in the top trace. 

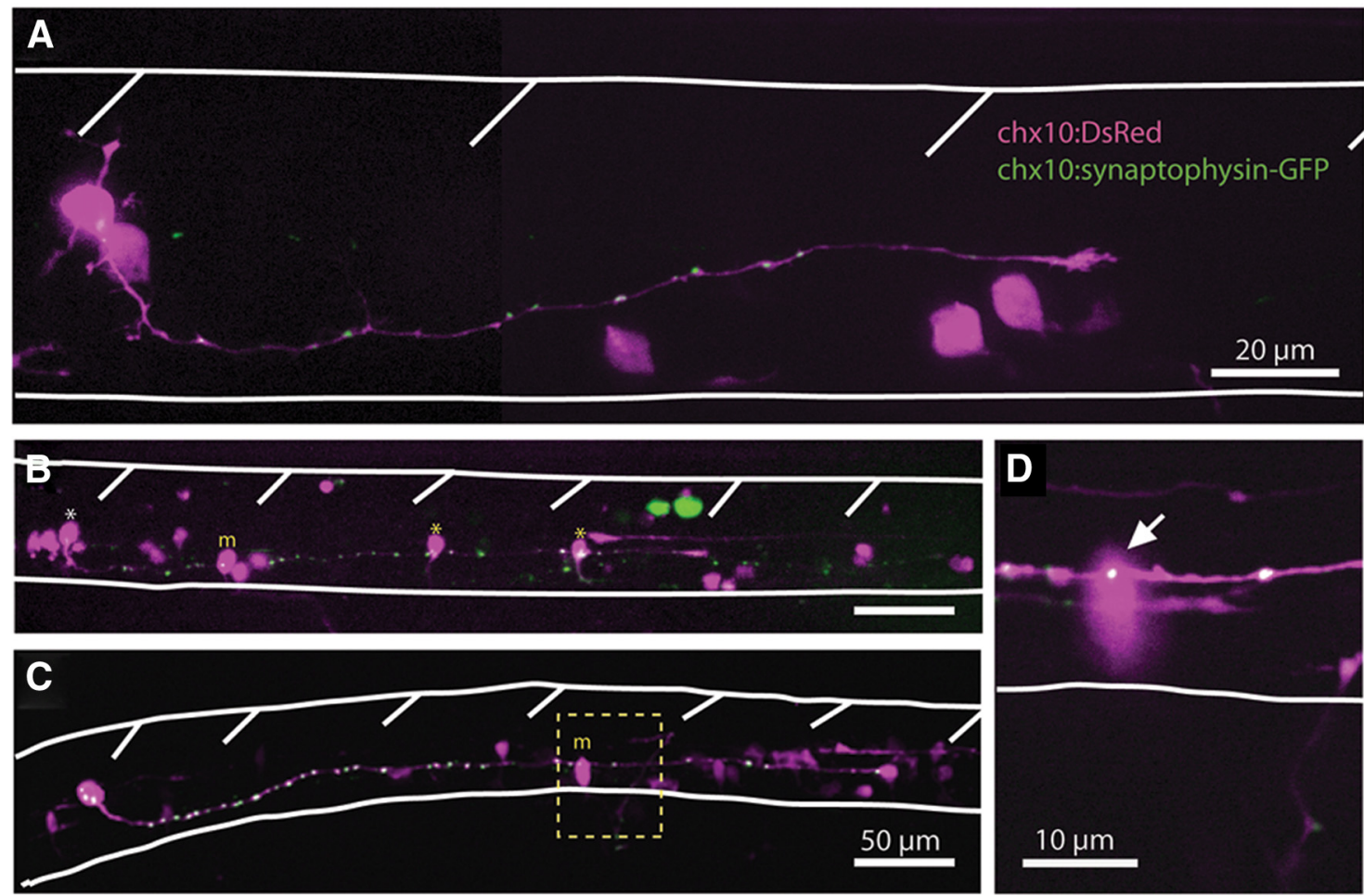

Figure 8. Embryonic spinal CiD neurons have putative synapses that contact caudal primary MNs and other CiDs. $A$, Representative example of a DsRed-expressing CiD neuron in a $24 \mathrm{hpf}$ embryo with distinct synaptophysin-GFP puncta along the length of the axon. Spinal cord and somite boundaries are outlined in white. B, A CiD neuron (white asterisk) in a $27 \mathrm{hpf}$ embryo has an axon spanning four somites and synaptophysin-GFP puncta that contact caudal CiDs (yellow asterisks) as well as a primary MN (yellow m). Scale bar, same as for C. C, A rostral CiD neuron in a 28 hpf embryo has an axon spanning $5-6$ somites and contacts a primary MN. D, High-resolution single $1 \mu \mathrm{m}$ confocal plane showing the putative synaptic contact (arrow) between the CiD and MN from the boxed area in $C$.

the most proximal part of the axon projecting from the soma, distinct synaptophysin-GFP puncta were visible along the entire length of the axon to the growth cone (Fig. 8A). CiDs with more dorsally located soma had the longest axons and the most synaptophysin-GFP puncta, which relates to the fact that these are the earliest-born CiDs (Kimura et al., 2006).

Older embryos had labeled CiDs with considerably longer axons and synaptic puncta (Fig. $8 \mathrm{~B}, \mathrm{C}$ ). Axons of CiD neurons in 27 hpf embryos spanned up to six somites in length and contained as many as 40 putative synaptic puncta. Several embryos had more than one cell expressing syp:GFP-DsR; and in addition to CiDs, primary MNs were also occasionally labeled (Fig. $8 B, C$ ), allowing for the identification of possible postsynaptic partners of CiD at these early ages. Synaptophysin-GFP puncta of CiD axons were seen to contact the soma of primary MNs as well as other, more caudal CiDs (Fig. $8 B-D$ ). These results show that, at the onset of double coiling, CiDs are extending axons that contact MNs and CiDs with putative glutamatergic synapses. Consequently, they are well suited to propagate ipsilateral excitation at early stages via glutamatergic neurotransmission.

\section{Discussion}

Double coiling: a novel intermediate behavior

The novel double coiling behavior described here has several characteristics that underlie its role as a transient, intermediate behavior that bridges the developmental gap between early single coiling with the onset of the touch response and the later appearance of swimming. The response to touch, which only slightly precedes the onset of double coiling, is a strong contraction contralateral to the site of touch, followed by one or two alternating coils (Saint-Amant, 2006). The touch response is, by definition, sensory-evoked and is glutamate-dependent, beginning with the activation of dorsal glutamatergic RB sensory neurons (SaintAmant, 2006; Pietri et al., 2009). However, RBs are not spontaneously active, therefore likely do not contribute to double coiling (Saint-Amant and Drapeau, 2001), in agreement with our evidence showing that touché mutants lacking mechanosensitive RBs (Low et al., 2010) show regular double coiling behavior.

The requirement of supraspinal connections for normal double coiling behavior but not for the response to touch (Pietri et al., 2009) suggests that at least part of the drive required for double coiling originates outside and therefore rostral to the spinal cord. By the onset of double coiling, axons of reticulopsinal neurons have projected to the rostral spinal cord (Mendelson, 1986) and may act upon the motor network via glutamatergic signaling to produce double coils. Because an increased $\mathrm{KCl}$ concentration enhanced double coiling in lesioned embryos, we hypothesize that hindbrain inputs may provide tonic drive to the rostral spinal cord at this age. If this were the case, the spinal cord should contain the minimal circuitry to generate double coiling but would require an external drive to bring the network to threshold to perform the behavior. The delayed onset of double coiling relative to the response to touch may simply be the result of a gradual buildup of the excitatory drive that becomes sufficient to spontaneously activate the network by $24 \mathrm{hpf}$ without the major touch-evoked release of glutamate from the RBs. Excitatory drive is likely to increase rapidly during development as intrinsic and synaptic properties of neurons are undergoing maturation, contributing to the transiency of the double coil behavior.

Double coiling is similar to previously described S-flexures observed in the salamander and in the chick (Hamburger, 1963) 
and may thus reflect a common vertebrate behavior. As in S-flexures, the contractions during a double coil overlap, such that the second contraction begins at the rostral end before the first contraction has terminated at the caudal end, implying a close interaction between the two sides of the spinal cord. This overlapping of contractions is also seen in swimming; therefore, double coiling is a logical intermediate to precede the lowamplitude, high-frequency swimming behavior. Swimming can be easily evoked but is more rarely spontaneously occurring in the embryo, perhaps because of a higher initial requirement for activation of the network compared with double coiling. However, once the threshold for swimming is reached, an immature burst can last several seconds in duration (Buss and Drapeau, 2001). In addition to the formation of chemical synapses, a shift in the activity of pacemaker IC cells toward more sustained burst firing may be important for the prolonged network activation underlying swimming (Tong and McDearmid, 2012).

\section{The integration of chemical neurotransmission into an existing electrical circuit}

Electrical coupling of neurons via gap junctions has been shown to contribute to the earliest activity in the developing nervous system of many invertebrate and vertebrate species, including mammals (Bennett and Zukin, 2004). The development of neurotransmission in the zebrafish spinal cord begins with an electrically coupled network solely driven by ipsilateral descending pacemaker activation from rostral spinal IC cells (Tong and McDearmid, 2012). For the circuit to be capable of mediating rapid double coiling, new chemical connections must be made that interconnect the two sides of the spinal cord to achieve alternating activation with greater control of timing and intensity than electrical coupling.

The earliest chemical synapses to form in the zebrafish spinal cord are glycinergic, starting at $20 \mathrm{hpf}$, followed a few hours later by glutamatergic synapses (Saint-Amant and Drapeau, 2000). GABA, the other major fast neurotransmitter, does not seem to play an important role in these circuits (Saint-Amant and Drapeau, 2000; Buss and Drapeau, 2001); therefore, glutamate and glycine appear to be the major chemical neurotransmitters driving behavior, as in other lower vertebrates (Grillner et al., 1991). Pharmacological blockade of glutamate receptors led to a significant reduction in double coiling behavior in the intact embryo as well as a loss of mixed events (correlates of double coiling) in MNs. In contrast, blockade of glycine receptors resulted in increased contralateral trunk contractions such that many double coils were transformed into triple and quadruple coils. These neurotransmitter systems may therefore play complementary roles whereby glutamate signaling drives the double coiling and glycinergic signaling acts to modulate the resulting excitation so as to prevent extended periods of quiescence resulting from circuit depression. Indeed, the latencies after these multiple-coil events were significantly longer than those after double coiling, suggesting that activity-dependent depression of network excitability occurs after coiling events of increasing strength and duration as seen in many developing vertebrate networks (O’Donovan, 1999).

\section{Neural activity patterns generating double coiling}

We propose that the physiological correlate of the double coiling behavior we are describing here, for the first time, corresponds at the cellular level to (in either order) a glycinergic synaptic burst (corresponding to a contralateral contraction) and gap junctiondriven periodic inward current (corresponding to an ipsilateral contraction) occurring in close, subsecond succession in the $\mathrm{MN}$. Glutamatergic events of increasing amplitudes become clearly integrated into the mixed event over the period of 24-29 hpf, suggesting the formation and/or maturation of glutamatergic synapses onto the MNs at this time. No other new patterns of activity appear in the $\mathrm{MN}$ during this time window until the onset of fictive swimming bursts at $28 \mathrm{hpf}$; therefore, the mixed events are likely correlates of double coiling.

Efficient contralateral activation of the spinal cord after an ipsilateral contraction could be achieved via excitatory interneurons that contact contralateral spinal $\mathrm{MNs}$ and premotor interneurons. During the period in which the circuit is capable of generating spontaneous single and double coils, however, the commissural glutamatergic CoPA neuron only fires a single action potential and then remains depolarized at the approximate reversal potential for chloride $(-35 \mathrm{mV})$ for $>1 \mathrm{~s}$, which is likely to shunt the membrane resistance and prevent further action potential firing. The CoPA is thought to be a sensory interneuron homologous to dorsolateral commissural sensory interneurons of Xenopus (Roberts and Sillar, 1990; Li et al., 2003; Saint-Amant, 2010) as they are both contacted by sensory RB neurons (Li et al., 2003; Pietri et al., 2009; Easley-Neal et al., 2013) and receive strong, shunting inhibition during evoked activity (Sillar and Roberts, 1992; Pietri et al., 2009). The role of the CoPA in spontaneous behavior is therefore likely in the response to inhibition rather than in triggering coils.

Our recordings of spontaneous activity in embryonic CiDs showed that, like MNs, these neurons receive synaptic glutamatergic activity at this time as well as electrical and glycinergic inputs that often reached threshold and resulted in the firing of several action potentials. Furthermore, CiDs showed activity patterns similar to mixed events in the ipsilateral MN and also fired action potentials during fictive double coiling, suggesting that they play an important role in this behavior. CiDs may be forming some of the earliest glutamatergic connections in the spinal cord. At $24 \mathrm{hpf}$, chx10+ cells (of which the CiD are the majority) are present at a density of 2 or 3 cells per hemisegment, and virtually all of these cells are positive for vglut2 at $32 \mathrm{hpf}$, the earliest time point examined (Kimura et al., 2006). Our mosaic labeling of syp:GFP-DSR in chx10+ cells showed that CiDs may be forming functional glutamatergic synapses as early as $24 \mathrm{hpf}$ onto MNs and other CiDs. Recent functional imaging studies using expression of a synaptophysin-GCaMP fusion protein have shown that calcium transients are seen at these puncta, suggesting that they are indeed functional presynaptic release sites (Nikolaou et al., 2012; Menelaou et al., 2014). These putative synaptic sites clearly colocalized with the soma of ipsilateral caudal MNs and CiDs and may be responsible for some of the glutamatergic synaptic events seen as early as $24 \mathrm{hpf}$ in our voltage-clamp recordings from CiDs and MNs.

In conclusion, spontaneous coiling in the embryo starts in an environment in which only gap junctions are present, creating a network of electrically coupled neurons, which can only propagate slow waves of activity. As the circuit matures, chemical synapses first add to the existing circuit without modifying the behavior too much, as we have shown with the transition from single to double coils. As development proceeds, the waning influence of gap junctions on circuit activity may lead to an opening for chemical synapses to finely sculpt local motor circuitry into producing faster and more intricate motor behaviors, such as swimming. 


\section{References}

Batista MF, Jacobstein J, Lewis KE (2008) Zebrafish V2 cells develop into excitatory $\mathrm{CiD}$ and Notch signalling dependent inhibitory VeLD interneurons. Dev Biol 322:263-275. CrossRef Medline

Ben-Ari Y (2002) Excitatory actions of gaba during development: the nature of the nurture. Nat Rev Neurosci 3:728-739. CrossRef Medline

Bennett MV, Zukin RS (2004) Electrical coupling and neuronal synchronization in the mammalian brain. Neuron 41:495-511. CrossRef Medline

Bernhardt RR, Chitnis AB, Lindamer L, Kuwada JY (1990) Identification of spinal neurons in the embryonic and larval zebrafish. J Comp Neurol 302:603-616. CrossRef Medline

Blankenship AG, Feller MB (2010) Mechanisms underlying spontaneous patterned activity in developing neural circuits. Nat Rev Neurosci 11:18-29. CrossRef Medline

Buss RR, Drapeau P (2001) Synaptic drive to motoneurons during fictive swimming in the developing zebrafish. J Neurophysiol 86:197-210. Medline

Chong M, Drapeau P (2007) Interaction between hindbrain and spinal networks during the development of locomotion in zebrafish. Dev Neurobiol 67:933-947. CrossRef Medline

Corner M (1978) Spontaneous motor rhythms in early life: phenomenological and neurophysiological aspects. Prog Brain Res 48:349-366. CrossRef Medline

Côté S, Drapeau P (2012) Regulation of spinal interneuron differentiation by the paracrine action of glycine. Dev Neurobiol 72:208-214. CrossRef Medline

Cui WW, Saint-Amant L, Kuwada JY (2004) shocked gene is required for the function of a premotor network in the zebrafish CNS. J Neurophysiol 92:2898-2908. CrossRef Medline

Cui WW, Low SE, Hirata H, Saint-Amant L, Geisler R, Hume RI, Kuwada JY (2005) The zebrafish shocked gene encodes a glycine transporter and is essential for the function of early neural circuits in the CNS. J Neurosci 25:6610-6620. CrossRef Medline

Dale N (1995) Experimentally derived model for the locomotor pattern generator in the Xenopus embryo. J Physiol 489:489-510. Medline

Downes GB, Granato M (2006) Supraspinal input is dispensable to generate glycine-mediated locomotive behaviors in the zebrafish embryo. J Neurobiol 66:437-451. CrossRef Medline

Drapeau P, Ali DW, Buss RR, Saint-Amant L (1999) In vivo recording from identifiable neurons of the locomotor network in the developing zebrafish. J Neurosci Methods 88:1-13. CrossRef Medline

Easley-Neal C, Fierro J Jr, Buchanan J, Washbourne P (2013) Late recruitment of synapsin to nascent synapses is regulated by Cdk5. Cell Rep 3:1199-1212. CrossRef Medline

Feller MB (1999) Spontaneous correlated activity in developing neural circuits. Neuron 22:653-656. CrossRef Medline

García-Colunga J, Miledi R (1999) Modulation of nicotinic acetylcholine receptors by strychnine. Proc Natl Acad Sci U S A 96:4113-4118. CrossRef Medline

Grillner S, Jessell TM (2009) Measured motion: searching for simplicity in spinal locomotor networks. Curr Opin Neurobiol 19:572-586. CrossRef Medline

Grillner S, Wallén P, Brodin L, Lansner A (1991) Neuronal network generating locomotor behaviour in lamprey. Annu Rev Neurosci 14:169-199. CrossRef Medline

Hale ME, Ritter DA, Fetcho JR (2001) A confocal study of spinal interneurons in living larval zebrafish. J Comp Neurol 437:1-16. CrossRef Medline

Hamburger V (1963) Some aspects of the embryology of behavior. Q Rev Biol 38:342-365. CrossRef Medline

Higashijima S, Schaefer M, Fetcho JR (2004) Neurotransmitter properties of spinal interneurons in embryonic and larval zebrafish. J Comp Neurol 480:19-37. CrossRef Medline

Kimmel CB, Ballard WW, Kimmel SR, Ullmann B, Schilling TF (1995) Stages of embryonic development of the zebrafish. Dev Dyn 203:253-310. CrossRef Medline

Kimura Y, Okamura Y, Higashijima S (2006) alx, a zebrafish homolog of
Chx10, marks ipsilateral descending excitatory interneurons that participate in the regulation of spinal locomotor circuits. J Neurosci 26:56845697. CrossRef Medline

Kimura Y, Satou C, Fujioka S, Shoji W, Umeda K, Ishizuka T, Yawo H, Higashijima S (2013) Hindbrain V2a neurons in the excitation of spinal locomotor circuits during zebrafish swimming. Curr Biol 23:843-849. CrossRef Medline

Knogler LD, Liao M, Drapeau P (2010) Synaptic scaling and the development of a motor network. J Neurosci 30:8871-8881. CrossRef Medline

Li WC, Soffe SR, Roberts A (2003) The spinal interneurons and properties of glutamatergic synapses in a primitive vertebrate cutaneous flexion reflex. J Neurosci 23:9068-9077. Medline

Low SE, Ryan J, Sprague SM, Hirata H, Cui WW, Zhou W, Hume RI, Kuwada JY, Saint-Amant L (2010) touché is required for touch-evoked generator potentials within vertebrate sensory neurons. J Neurosci 30:9359-9367. CrossRef Medline

McDearmid JR, Drapeau P (2006) Rhythmic motor activity evoked by NMDA in the spinal zebrafish larva. J Neurophysiol 95:401-417. CrossRef Medline

Mendelson B (1986) Development of reticulospinal neurons of the zebrafish: II. Early axonal outgrowth and cell body position. J Comp Neurol 251:172-184. CrossRef Medline

Menelaou E, VanDunk C, McLean DL (2014) Differences in spinal V2a neuron morphology reflect their recruitment order during swimming in larval zebrafish. J Comp Neurol 522:1232-1248. CrossRef Medline

Meyer MP, Smith SJ (2006) Evidence from in vivo imaging that synaptogenesis guides the growth and branching of axonal arbors by two distinct mechanisms. J Neurosci 26:3604-3614. CrossRef Medline

Narayanan CH, Fox MW, Hamburger V (1971) Prenatal development of spontaneous and evoked activity in the rat (Rattus norvegicus albinus). Behaviour 40:100-134. CrossRef Medline

Nikolaou N, Lowe AS, Walker AS, Abbas F, Hunter PR, Thompson ID, Meyer MP (2012) Parametric functional maps of visual inputs to the tectum. Neuron 76:317-324. CrossRef Medline

O'Donovan MJ (1999) The origin of spontaneous activity in developing networks of the vertebrate nervous system. Curr Opin Neurobiol 9:94104. CrossRef Medline

Pietri T, Manalo E, Ryan J, Saint-Amant L, Washbourne P (2009) Glutamate drives the touch response through a rostral loop in the spinal cord of zebrafish embryos. Dev Neurobiol 69:780-795. CrossRef Medline

Roberts A, Sillar KT (1990) Characterization and function of spinal excitatory interneurons with commissural projections in Xenopus laevis embryos. Eur J Neurosci 2:1051-1062. CrossRef Medline

Saint-Amant L (2006) Development of motor networks in zebrafish embryos. Zebrafish 3:173-190. CrossRef Medline

Saint-Amant L (2010) Development of motor rhythms in zebrafish embryos. Prog Brain Res 187:47-61. CrossRef Medline

Saint-Amant L, Drapeau P (1998) Time course of the development of motor behaviors in the zebrafish embryo. J Neurobiol 37:622-632. CrossRef Medline

Saint-Amant L, Drapeau P (2000) Motoneuron activity patterns related to the earliest behavior of the zebrafish embryo. J Neurosci 20:3964-3972. Medline

Saint-Amant L, Drapeau P (2001) Synchronization of an embryonic network of identified spinal interneurons solely by electrical coupling. Neuron 31:1035-1046. CrossRef Medline

Sillar KT, Roberts A (1992) Phase-dependent modulation of a cutaneous sensory pathway by glycinergic inhibition from the locomotor rhythm generator in Xenopus embryos. Eur J Neurosci 4:1022-1034. CrossRef Medline

Tong H, McDearmid JR (2012) Pacemaker and plateau potentials shape output of a developing locomotor network. Curr Biol 22:2285-2293. CrossRef Medline

van Mier P, Armstrong J, Roberts A (1989) Development of early swimming in Xenopus laevis embryos: myotomal musculature, its innervation and activation. Neuroscience 32:113-126. CrossRef Medline 